\title{
Exploring New Qualitative Methods to Support a Quantitative Analysis of Glance Behavior
}

\author{
Mauricio Muñoz ${ }^{1,2,3}$, Bryan Reimer ${ }^{1}$, Bruce Mehler ${ }^{1}$ \\ ${ }^{1}$ MIT AgeLab \& New England University Transportation Center, Cambridge, Massachusetts, USA \\ ${ }^{2}$ TU Munich, LMU Munich, Munich, Germany \\ ${ }^{3}$ University of Augsburg, Augsburg, Germany \\ ammd@mit.edureimer@mit.edu bmehler@mit.edu
}

\begin{abstract}
The work proposes conceptual ways of considering drivers' visual behavior over time as a flow of attention across space. These efforts extend upon previous works (research and regulatory) that focus in greater detail on quantifying attention independent of time. The primary contribution of this work lies in the presented methodology for analysis of glance allocation features. An application of this method is explored as an illustration of its analytic potential. Driver glance allocation data were drawn from a heterogeneous set of drivers. Double coded and mediated glance allocations were used to detail differences between drivers' visual behavior across 3 different task types (baseline driving, visual-manual interaction, auditory-vocal interaction). Glance transition counts were used to estimate glance transition probabilities and significance values. Glance duration features were explored in parallel and used to visualize temporal allocation distribution and significance. Results show that visualization techniques based on these features are well suited for qualitative analysis of visual attention.
\end{abstract}

\section{Author Keywords}

Data visualization; driving; task modality; driver glance behavior; visual demand; time series.

\section{ACM Classification Keywords}

H.5.2 Information interfaces and presentation (e.g. HCI): User Interfaces.

\section{INTRODUCTION}

Driving is a multifaceted activity that places the driver under a wide array of physical and cognitive demands. Visual demand is one such type of workload that has long been shown to correlate with safety [31]. Drivers' glance allocation strategies - visual scan patterns - have been understood to vary with the type of task ([40], [11], [20],

Permission to make digital or hard copies of part or all of this work for personal or classroom use is granted without fee provided that copies are not made or distributed for profit or commercial advantage and that copies bear this notice and the full citation on the first page. Copyrights for thirdparty components of this work must be honored. For all other uses, contact the Owner/Author. Copyright is held by the owner/author(s). AutomotiveUI '15, September 01-03, 2015, Nottingham, United Kingdom (C) 2015 ACM. ISBN 978-1-4503-3736-6/15/09 ..\$15.00

DOI:http://dx.doi.org/10.1145/2799250.2799278
[12], [34]). Works such as [5], [33], [22], [29], [21], [31], [30] suggest that that adding auditory-vocal options to interfaces can decrease the total visual demand required to complete an activity. Changes in driver glance allocation behavior across the operating environment have been shown to be sensitive to changes in cognitive demands and task complexity ([25], [36], [39], [37], [38], [5], [31], [33]).

Efforts that have utilized the allocation of visual attention to infer cognitive demands illustrate that a driver's visual scanning behavior may be a valuable workload indicator. In one of the primary measures for driver attentiveness ([2], [8], [38], [7]) - clearly, visual attention allocation is a relevant subject of study due to the positive correlation that exists between off-road glances and crashes and near crash events. This has been explored in a more general predictive context ([19], [41], [28], [16]).

The growing popularity of voice interfaces in vehicles (e.g. [14]) represents a move away from more traditional visualmanual driver vehicle interaction paradigms. There is substantial empirical evidence that suggests that visualmanual and speech-based interaction modalities result in intrinsically different driver demand profiles [24]. In comparison to speech-based interactions, visual-manual tasks generally result in greater impairment in driving performance ([13], [31], [21]), have an impact on detection response tasks ([23], [3]), and pose a heightened risk of crash-related events ([8], [17], [38]).

The motivation behind this report is to provide a comprehensive way to qualitatively assess patterns in drivers' visual scanning strategies. Its principal contribution lies in the presented methodology for analysis of visual attention features, presenting simple statistical procedures tailored to glance allocation features in the driving scenario. These techniques were developed as an initial stage in a wider predictive analysis context that draws upon other features (see [24]). They are therefore designed to be used as supplemental analysis tools, providing a visual guide to aid the discovery of prominent patterns that may lie in the data. They act as a support mechanism, e.g. in the context of interface development, allowing for instance a visual assessment of the degree to which a vehicle's interface impacts driver attention allocation. 


\section{Related work}

Driver scan patterns have an established history as behavioral features, particularly in the context of driver safety analysis ([40], [5], [19], [38], [32], [34]). Most if not all of these reports consider glance features in an aggregated form or gaze measurements derived from an eye tracking system, providing low-level quantitative measures of driver demand based on visual allocation over time and/or space. A comprehensive review of the literature in this area is beyond the scope of this paper, however a number of illustrative studies are provided as a general overview.

Glance duration has long been recognized as a significant input for quantifying driver visual behavior. [9] explore visual demands of vehicle navigation systems in terms of mean glance time and glance histograms per task. Aggregates over time (i.e. eyes-off-road time, on-road-tasktime) are recommended as a primary safety measure. [8] quantify driver performance when using a cell-phone using features such as percent of Total-Eyes-Off-Road Time (TEORT) or Total-Eyes-Off-Roadway Time ([17], [26]).

Some reports have focused on glance location as a sole descriptor. [7], [27] analyze drivers' glance fixations in the scene in order to identify glance allocations that reduce the likelihood of an accident among novice drivers. [40], [32], [34] are among studies that have analyzed gaze dispersion statistics as a means of inferring a change in the driver's visual behavior with varying workload levels. [40] critiques the power of the percent road center (PRC) metric as employed by other reports (e.g. [6], [37], [12]).

Intuitively, it is clear that analyzing visual behavior along both temporal and spatial dimensions may yield additional benefits. [38] explore the relationship between glance behavior histories during secondary tasks and crash risk. The report proposes metrics for identifying high-risk patterns (with a focus on lead vehicle closing), expanding on the TEORT duration in favor of the Total Eyes off Path Time (TEOPT) variable as a potential indicator of high crash risk. [19] define location, duration, and history as the three principal characteristics of eye patterns. The report looks at using these features as inputs to hand-crafted, lowlevel functions in order to approximate driver distraction. Statistical decision procedures such as maximum Odds Ratio or $\mathrm{R}^{2}$ are employed in order to predict crash and nearcrash risk. [16] illustrate the concept of a visual attention buffer which to an extent encodes both glance duration and duration information, providing a dynamic representation of the driver's attention profile over time. [25] look at driver response behavior by using a response task during baseline vs. hands-free phone dialing tasks. Their experiments focus on glance fixation coordinates and duration to assess spatial gaze variability per subject and task. In a study that finds a similar gaze concentration effect with rising workload levels, [37] address the shortcoming of traditional descriptions of glance behavior and use glance frequency to build fixation duration histograms and gaze concentration profiles per task. A variety of other studies ([18], [36], [12]) look at both glance frequency and duration data as a measure of visual demand.

In short, approaches have focused so far on providing aggregate summaries of glance location and duration over time as separate facets of drivers' visual behavior. These reports have largely focused on presenting quantified data in the form of classical statistical measures. This report extends on previous work in a number of ways. First, it thinks beyond the concept of traditional gaze features, exploring glance transition data ([1], [4], [35]) as a key indicator of driver behavior. More importantly, it provides a unified method for visualizing glance behavior across both time and space, allowing an inexpensive, high level exploration of visual behavior as stepping stone for further quantitative research.

\section{Data}

Data were taken from an on-road study conducted at the MIT AgeLab designed to assess the properties of production voice interfaces ([22], [30]). Data from 96 subjects collected in a 2010 vehicle and evenly spread among 4 age groups (20-24, 25-39, 40-54, and 55+) were considered. Subjects completed a wide array of tasks, including 2 "hard" radio tuning tasks using both the vehicle's manual and voice interfaces. The manual interface required a button press, selecting a touch screen button, and rotating a dial to a specified station. "Voice" tuning included initial manual selection of a push-to-talk button on the steering wheel. Two minute baseline periods of just driving preceded each task type. Additionally, subjects were equally divided across 3 subgroups. The first received guided training on each task prior to on-road assessment. The second was informed of the format of the tasks they would be asked to do on-road and given the opportunity to practice, but were otherwise self-trained. The final subgroup completed the tasks using the vehicle's voice interface in "expert" mode, where most prompting commands and yes/no confirmations were removed.

For each subject and task period, glance allocations were extracted by two coders and mediated by a third (see [29] for methodological details). Glance allocations (location, timestamp) were binned to a total of 11 possible locations in the vehicle (see axes on Figures 3 and 4). The "Unknown" and "Other" locations were reserved for problematic allocations due to factors such as video quality or glare. Timestamps were used to extract the duration of each glance by computing the absolute time difference between a given allocation and the next. These data were then organized into a hierarchical time series of glances per subject, task modality, and task, using mediated glance annotations where possible. 


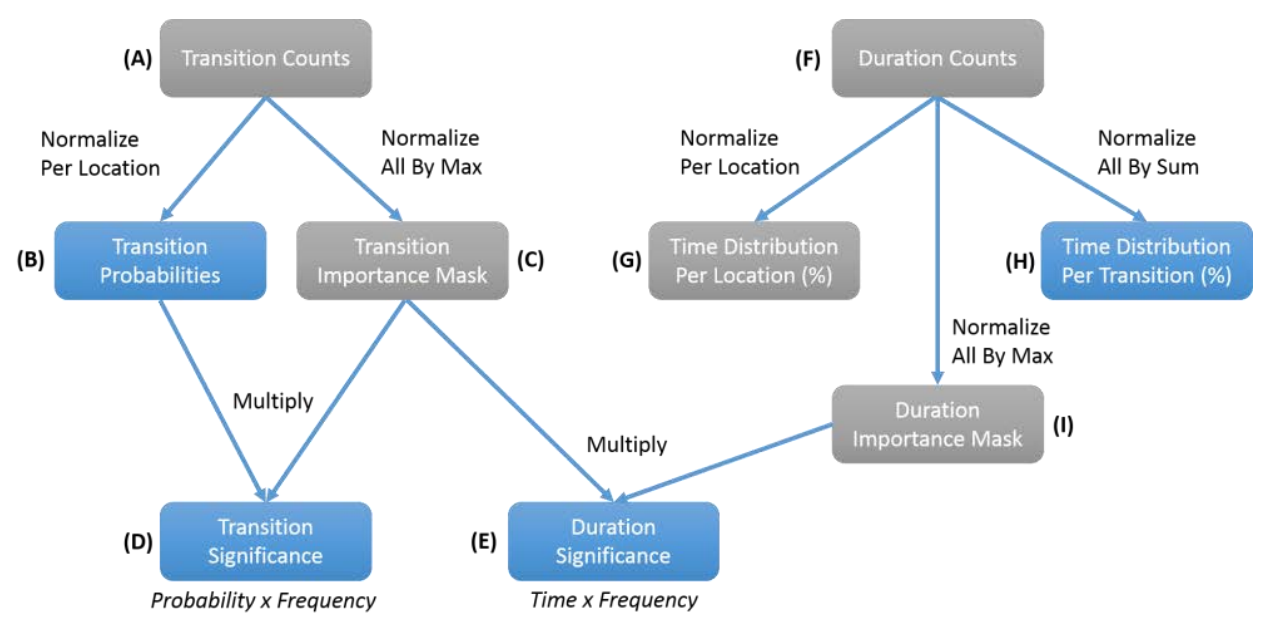

Figure 1. Glance behavior visualization strategies. Nodes represent data visualizations and arrows detail data operations. Illustrative results are presented for blue nodes.

\section{METHODS}

Figure 1 provides a methodological summary of the visualizations and analyses considered. Transition counts (A) are extracted from the annotated data, illustrating how often the time series of glance data progresses from an allocation at location $\mathrm{J}$ to location $\mathrm{K}$ for all location pairs $\mathrm{J}$, $\mathrm{K}$, which fall within the 11 aforementioned discrete locations. Normalizing these values (B) for each location (row) as follows yields a transition probability matrix $P($ current glance $=K \mid$ previous glance $=J)$ : given a subject's set of glances organized as a time series for a particular task, count the number of transitions from location $\mathrm{J}$ to location $\mathrm{K}(\mathrm{A})$, then divide by the total number of transitions out of location J. This concept is illustrated in Figure 2, which shows transition probabilities to and from the forward roadway as a heat map across all drivers during baseline driving (driving with no secondary activity).

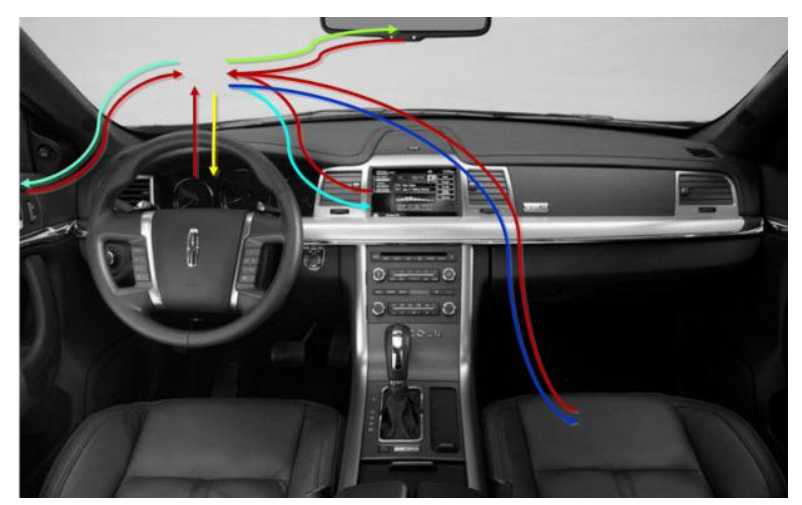

Figure 2. Glance transition probabilities to and from the forward roadway across the entire subject pool visualized as heat transitions overlaid on a vehicle cab during driving without the presence of any experimental secondary activities.
These matrices (Figure 1B) represent the probability of transitioning from a given location to another. They do not, however, consider the number of glances used to produce the probability estimate. This factor limits the overall potential for comparison, leading to difficulties in interpretation across matrices or between different locations within a given matrix (i.e. a single glance transition associated with an infrequently visited region of interest can result in a high transition probability, while regions frequently visited will represent a more stable probability prediction). In order to account for this limitation, a complementary representation was developed. Transition counts (A) were first normalized to fit the range [0, 1], resulting in a transition importance mask (C). Using element-wise multiplication, this mask was brought together with the transition probabilities (B). The result is a transition significance matrix (D), where high-intensity entries mark transitions that are not only probable, but that also appear often in the underlying data. In contrast to (B), the rows of this matrix must not necessarily sum to 1 .

This analysis only takes the number of transitions into account, without reflecting the fact that each glance movement implicitly carries an associated duration value. Semantically, the duration of a transition from glance location $\mathrm{K}$ to glance location $\mathrm{J}$ may be defined as the duration of the glance at location $\mathrm{K}$ before switching to glance location J. Matrix (F) represents this result for all locations J, K. Previously, each transition was weighed equally (individual transition types were counted). This approach can be viewed as weighing each transition with the magnitude of its duration. Normalizing the resulting matrix for each glance location (as in the step from A to B) yields the distribution of task time (G) spent across all locations as a function of the glance object (rows necessarily sum to 1 ). On the other hand, normalizing by the sum of all entries in the matrix describes what 
percentage of the total time across all tasks and subjects was spent in each type of glance movement $(\mathrm{H})$. The sum of all elements in this matrix is 1 .

This matrix $(\mathrm{H})$ does not however encode whether large amounts of time allotted to a particular movement type are the result of few, long duration glances, or of many short duration glances. In order to highlight the difference, a duration importance mask was computed by globally normalizing all duration count values to unity (I). Using simple element-wise multiplication, this mask may be combined with the transition importance mask $(\mathrm{C})$, yielding a duration significance map where high intensity values correspond to long duration glances that also appear frequently in the data $(\mathrm{E})$. This last step reveals additional patterns: a switch from high to low intensity is indicative of infrequent, long-duration glances. A switch from low to high intensity is suggestive of frequent, short-duration glances.

This visual modeling approach affords the researcher a simple way to intuitively compare the patterns summarized in these matrices. Individual transition probability (B) and significance matrices (D) encode how the average driver allocates visual attention during a period of interest. Similarly, matrices $(\mathrm{E})$ and $(\mathrm{H})$ describe how the average driver distributes attention on a temporal dimension. Any two such matrices (i.e. corresponding to different in-vehicle tasks, age groups, genders, vehicles, environments etc.) may be easily compared using element-wise subtraction, and visualized using e.g. a two-scale intensity scheme, where areas of higher intensity encode category-specific salient visual behavior patterns.

\section{RESULTS}

While the goal of this work is to introduce a methodology tailored to the analysis of gaze data, an effort is made to concretize its significance by providing an illustrative example using real-world driver gaze data. Results are presented in the form of derived matrices at every stage of this analysis in order to visualize differences between task modalities (baseline driving, visual-manual radio tuning, and auditory-vocal radio tuning). Figure 3 illustrates transition probability matrices (Figure 1B) for driving without the presence of any experimental secondary activities (baseline driving - top), visual-manual radio tuning (middle), and auditory-vocal radio tuning (bottom). As expected, the manual radio tuning tasks clearly show a strong shift in attention from the roadway to the center stack. In contrast, the spatial distribution of visual attention for voice-based radio tuning tasks illustrates that there is a high percentage of transitions from the road to a broad number of regions including the center stack as well as other awareness-relevant locations. In short, the allocation of visual attention during auditory-vocal radio tuning tasks more closely mirrors that of baseline driving. Given that both activities draw upon modest amounts of total off-road glance times, this raises the question as to how well metrics such as total off-road glance time or glance time to the device represent the relative demand (and conceivably safety impact) of these tasks. This visualization clearly shows that even during voice-based activities, the center stack is still a highly salient object of interest when looking away from the forward roadway. Figure 3 (bottom) illustrates a limitation: transitions from "Other" to center stack are visualized with a high intensity although the number of such transitions is very low (there are a total of only 9 transitions starting at "Other").

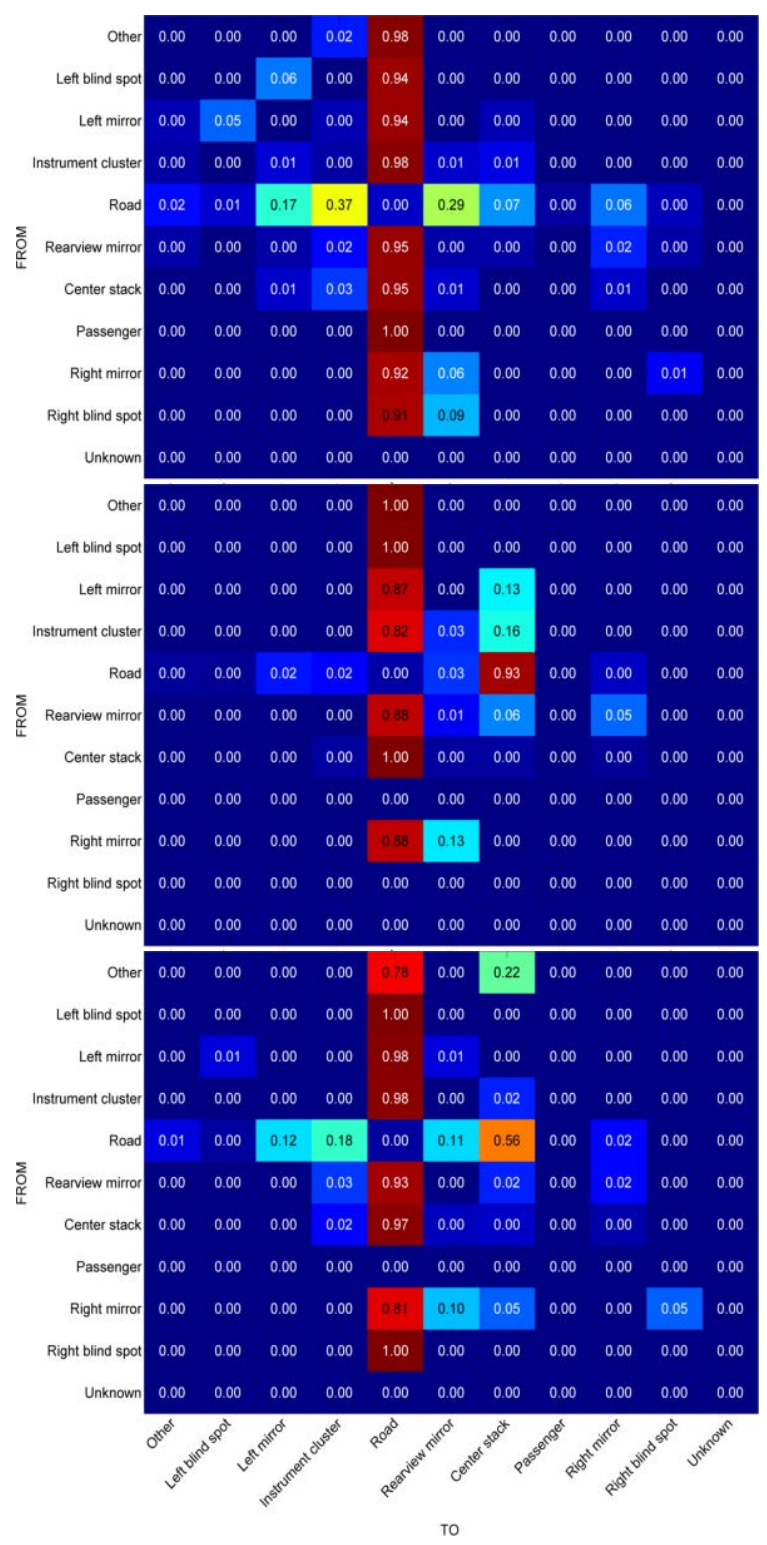

Figure 3. Transition probability matrices (B): (top) baseline driving, (middle) visual manual, (bottom) auditorv-vocal radio task

The computation of transition significance matrices (Figure 1D) is illustrated in Figure 4, column 1. This approach mitigates the influence of infrequent transitions. High intensity entries serve as a combined representation of transitions that are both probable and frequent. Considering 
the frequency of a given transition reduces noise in the visualization and interpretation of attention allocation; only transitions that significantly model the average driver's visual behavior are displayed on a scale of relative importance.

The three significance matrices in column 1 further verify the relative divergence in attention allocation between baseline driving (top row) and visual-manual interaction (middle row). A comparison of the auditory-vocal tasks (bottom row) to baseline driving further highlights a general degree of similarity given the presence of frequent glances that support driver awareness (i.e. to mirrors). Not surprisingly, visual behavior during visual-manual interactions may essentially be reduced to transitions between the center stack and the forward roadway. Another powerful result indicates slightly more significant transitions from the center stack to the road than transitions from the road to the center stack. This may very well be indicative of the driver's risk perception of a visual-manual task and engrained motivation to remain aware of the operating environment. This exchange between the center stack and forward roadway is also found under auditoryvocal load (Figure 4, column 1, bottom).

Taking glance durations into account extends the methodology to the temporal domain (Figure 4, column 2). As illustrated in the top row, drivers' attention was almost wholly allocated to awareness-relevant regions during baseline driving. Visual-manual tuning (middle row) diverts attention so that $93 \%$ of total task time is spent on glances between the forward roadway and the center stack. This effect is alleviated in the auditory-vocal case (bottom row) where $74 \%$ of the total task time was spent on forward roadway glances. Fixing gaze to the forward roadway and then glancing to the center stack accounts for $39 \%$ of the total task time. This is a considerably higher percentage than the corresponding value for glances to the center stack followed by a glance to the road (only 15\% of total task time). This observation gives a clear illustration of the intrinsic differences between the present visual-manual and auditory-vocal radio tuning data: the center stack clearly holds a priority in the drivers' attention management in the former case. This, however, does not hold for the auditoryvocal case, where drivers clearly prioritize the forward roadway in spite of semi-frequent glances to the center stack.

Column 2 in Figure 4 considers the percentages of total task time; the 3rd column illustrates the concept of a duration significance matrices (E). A clear inverse relationship may be observed between the significance levels of the center stack to road and road to center stack glance movements for the visual-manual and auditory-vocal radio tuning tasks. Concretely, even after considering how frequent these transition types are in the data, it is clear that these visualmanual tasks exhibit a strong tendency towards the center stack to road movement, while their auditory-vocal counterparts encourage a more distributed allocation of visual attention. This provides evidence that the present instance of the auditory-vocal tasks permit drivers to maintain some level of attention to broader aspects of the operational task.

\section{DISCUSSION AND FUTURE WORK}

The available literature often presents quantitative summaries of driver glance behavior in the form of aggregated statistics over time and the operating space. There appears to be more limited effort invested in visualization methods (e.g. [10], [15]) designed specifically for its qualitative analysis. This report extends previous efforts in modeling driver glance behavior by providing a multifaceted and predominantly visual representation of glance allocation strategies on temporal and spatial dimensions, both independently and jointly. This report focuses on exploring visual behavior not in terms of individual glances, but rather looks at patterns beyond static allocations to show how they are interrelated. There are limitations, however. The approach restricts itself to chains of 2 visual targets, as adding intermediary elements would most likely convolute the visualization beyond use (i.e. jump from $2 \mathrm{D}$ matrices to $3 \mathrm{D}$ cubes for 3 targets). The approach does not, however, require glances to be neighbors in time, allowing for the analysis of glance transitions on any given temporal scale (i.e. "sample rate" of glance allocations). Additionally, static glance regions are required for a meaningful comparison between individual matrices. The approach does not, however, define how these regions must be defined, i.e. dynamically changing targets may be handled by clustering them according to some predefined grouping criterion (e.g. awareness-related vs. non awareness-related glances).

In order to help the reader concretize the potential gains of the main contribution of this work, glance data were split across task modalities and pushed through the analytic pipeline in Figure 1. The relationship between baseline driving and driving with a subtask load of an auditory-vocal kind becomes especially interesting as voice interfaces gain in popularity. Similarities are revealed in terms of the distribution of awareness-related glances. Comparing indicators for visual-manual vs. auditory-vocal radio tuning shows differences, i.e. in how the center stack is prioritized.

This type of analysis may be thought of as an early stage for more intricate, quantitative modeling work. Divergence matrices are not included in the present results due to space limitations (see [24]).

\section{CONCLUSION}

The present work examines the relationships between glances over time as primary visual behavior features. A hierarchy of analytic methods tailored to these features is presented. Transition probability and significance matrices encode how drivers, on average, allocate visual attention. Complementing visualizations describe visual behavior in terms of glance duration. An illustrative application of this methodology is presented using annotated driver glance data. 


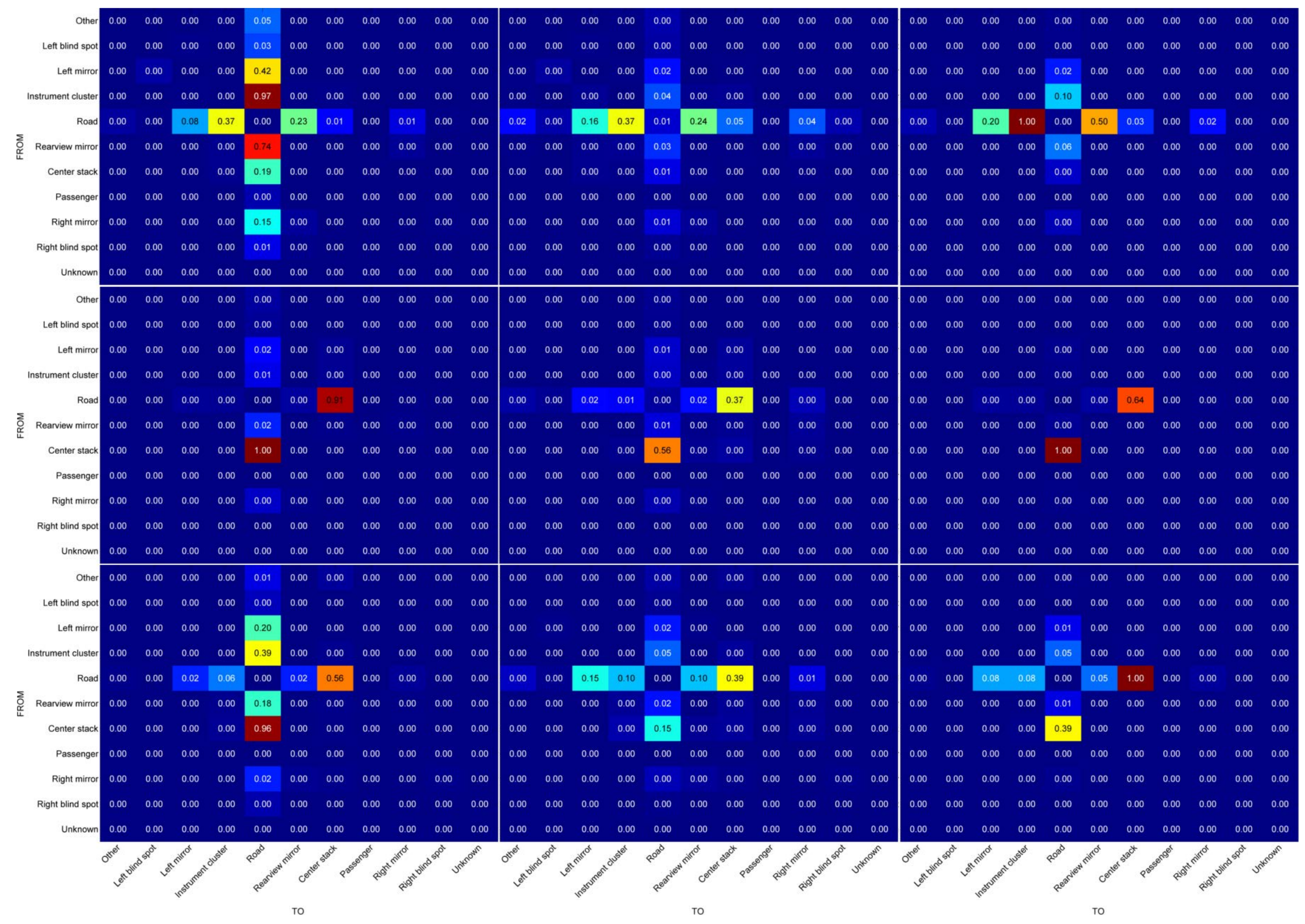

Figure 4. Transition matrices "from" and "to" defined locations where column: $1=$ transition significance $(D), 2=$ time distribution per transition $(H), 3=$ duration significance (E). Rows: (top) baseline driving, (middle) visual-manual radio tuning, (bottom) auditory-vocal radio tuning. 
The results show qualitative differences among three key task types (baseline driving, visual-manual, and auditoryvocal radio tuning). Concretely, interactions during visualmanual radio tuning may essentially be reduced to repeated transitions to and from the center stack. During the auditory-vocal radio tuning tasks, attention to peripheral locations was maintained in spite of strong engagement with the center stack. Long duration glances to the center stack followed by a transition to the forward roadway are more characteristic of the visual-manual radio tuning data, whereas the auditory-vocal tasks clearly manifested the opposite. The techniques presented in this report may be considered as an early feasibility analysis for further modeling initiatives along any given dimensions of the underlying data at hand.

\section{ACKNOWLEDGEMENTS}

Support for the initial development of this work was provided by the Advanced Human Factors Evaluator for Automotive Demand (AHEAD) Consortium. Further support was provided by the US DOT's Region I New England University Transportation Center at MIT and the Toyota Class Action Settlement Safety Research and Education Program. The views and conclusions being expressed are those of the authors, and have not been sponsored, approved, or endorsed by Toyota or plaintiffs' class counsel. Acknowledgement is also extended to The Santos Family Foundation and Toyota's CSRC for providing funding for the studies [20], [28] from which the data were drawn.

\section{REFERENCES}

1. Ahlstrom, C., Kircher, K., Thorslund, B., \& Adell, E. (2015). Bicyclists' visual strategies when conducting self-paced vs. system-paced smartphone tasks in traffic. Transportation Research Part F (0). doi: http://dx.doi.org/10.1016/j.trf.2015.01.010

2. Bach, K. M., Jæger, M. G., Skov, M. B., \& Thomassen, N. G. (2009, September). Interacting with in-vehicle systems: understanding, measuring, and evaluating attention. In Proceedings of the 23rd British HCI Group Annual Conference on People and Computers (pp. 453-462). British Computer Society.

3. Beckers, N., Schreiner, S., Bertrand, P., et al. (2014, September). Comparing the Demands of Destination Entry using Google Glass and the Samsung Galaxy S4. In Proceedings of the Human Factors and Ergonomics Society Annual Meeting (No. 1, pp. 2156-2160).

4. Birrell, S. A., \& Fowkes, M. (2014). Glance behaviours when using an in-vehicle smart driving aid: A realworld, on-road driving study. Transportation Research Part F 22(0), 113-125. doi: http://dx.doi.org/10.1016/j.trf.2013.11.003

5. Chiang, D.P., Brooks, A.M., \& Weir, D.H. (2005). Comparison of visual-manual and voice interaction with contemporary navigation system HMIs (2005-010433). SAE 2005 World Congress \& Exhibition.

6. Engström, J., Johansson, E., \& Östlund, J. (2005). Effects of visual and cognitive load in real and simulated motorway driving. Transportation Research Part F, 8(2), 97-120.

7. Fisher, D. L., Pollatsek, A. P., \& Pradhan, A. (2006). Can novice drivers be trained to scan for information that will reduce their likelihood of a crash? Injury Prevention, 12(suppl 1), i25-i29.

8. Fitch, G. A. S., S.A., Guo, F., McClafferty, J., Fang, Y., Olson, R. L., Perez, M. A., et al. (2013). The impact of hand-held and hands-free cell phone use on driving performance and safety-critical event risk (DOT HS 811 757). Washington, DC.

9. Green, P. (1999). Visual and task demands of driver information systems (UMTRI-98-16,).

10. Gotz, D., \& Wen, Z. (2009, February). Behavior-driven visualization recommendation. In Proceedings of the 14th international conference on Intelligent user interfaces (pp. 315-324). ACM.

11. Haigney, D.E., Taylor, R.G., \& Westerman, S.J. (2000). Concurrent mobile (cellular) phone use and driving performance: task demand characteristics and compensatory processes. Transportation Research Part F, 3, 113-121.

12. Harbluk, J. L., Noy, Y. I., Trbovich, P. L., \& Eizenman, M. (2007). An on-road assessment of cognitive distraction: Impacts on drivers' visual behavior and braking performance. Accident Analysis \& Prevention, 39(2), 372-379.

13. He, J., Chaparro, A., Nguyen, B., et al. (2013, October). Texting while driving: is speech-based texting less risky than handheld texting?. In Proceedings of the 5th International Conference on Automotive User Interfaces and Interactive Vehicular Applications (pp. 124-130). ACM.

14. IHS Technology. 2013. Voice Recognition Installed in More than Half of New Cars by 2019. (19th March 2013). Retrieved 12th April, 2015 from https://technology.ihs.com/427146/

15. Keim, D. A. (2002). Information visualization and visual data mining. IEEE Transactions on Visualization and Computer Graphics, 8(1), 1-8.

16. Kircher K., Ahlström C. (2009, September). Issues related to the driver distraction detection algorithm AttenD. Paper presented at the 1st International Conference on Driver Distraction and Inattention, Gothenburg, Sweden.

17. Klauer, S. G., Guo, F., Simons-Morton, B. G., et. al. (2014). Distracted driving and risk of road crashes among novice and experienced drivers. New England Journal of Medicine, 370(1), 54-59. 
18. Lansdown, T. C. (1996). Visual demand and the introduction of advanced driver information systems into road vehicles (Doctoral dissertation).

19. Liang, Y., Lee, J.D., \& Yekhshatyan, L. (2012). How dangerous is looking away from the road? Algorithms predict crash risk from glance patterns in naturalistic driving. Human Factors, 54(6), 1104-1116.

20. Maciej, J. \& Vollrath, M. (2009). Comparison of manual vs. speech-based interaction with in-vehicle information systems. Accident Analysis and Prevention, 41, 924-930.

21. Mehler, B., Kidd, D., Reimer, B., Reagan, I., Dobres, J. $\&$ McCartt, A. In Press. Ergonomics. Multi-modal assessment of on-road demand of voice and manual phone calling and voice navigation entry across two embedded vehicle systems. Arlington, VA: Insurance Institute for Highway Safety.

22. Mehler, B., Reimer, B., Dobres, J., et al. (2014). Further evaluation of the effects of a production level "voice-command" interface on driver behavior: replication and a consideration of the significance of training method. 2014-2. Massachusetts Institute of Technology, Cambridge, MA.

23. Munger, D., Mehler, B., Reimer, B., et al. (2014, September). A Simulation Study Examining Smartphone Destination Entry while Driving. In Proceedings of the 6th International Conference on Automotive User Interfaces and Interactive Vehicular Applications (pp. 1-5). ACM

24. Muñoz, M. (2015). Modeling Driver Behavior Using Hidden Markov Models. TU Munich, LMU Munich, University of Augsburg (Manuscript in preparation).

25. Nunes, L., \& Recarte, M. A. (2002). Cognitive demands of hands-free-phone conversation while driving. Transportation Research Part F 5(2), 133-144.

26. Olson, R. L., Hanowski, R. J., Hickman, J. S., \& Bocanegra, J. L. (2009).Driver distraction in commercial vehicle operations (FMCSA-RRR-09042).

27. Pradhan, A. K., Hammel, K. R., DeRamus, R., et al. (2005). Using eye movements to evaluate effects of driver age on risk perception in a driving simulator. Human Factors, 47(4), 840-852.

28. Pohl, J., Birk W., Westervall L. (2007). A driverdistraction-based lane-keeping assistance system. Proceedings of the IMechE Part I: Journal of Systems and Control Engineering, 221, 541-552

29. Reimer, B., Mehler, B., Dobres, J. \& Coughlin, J.F. (2013). The effects of a production level "voicecommand" interface on driver behavior: reported workload, physiology, visual attention, and driving performance. 2013-17A. Massachusetts Institute of Technology, Cambridge, MA.
30. Reimer, B., Mehler, B., Dobres, J., McAnulty, H., Mehler, A., Munger, D., \& Rumpold, A. (2014, September). Effects of an 'Expert Mode' Voice Command System on Task Performance, Glance Behavior \& Driver Physiology. In Proceedings of the 6th International Conference on Automotive User Interfaces and Interactive Vehicular Applications (pp. 1-9). ACM.

31. Reimer, B., Mehler, B., Reagan, I, Kidd, D., \& Dobres, J. (2015). Multi-modal demands of a smartphone used to place calls and enter addresses during highway driving relative to two embedded systems. Arlington, VA: Insurance Institute for Highway Safety.

32. Reimer, B., Mehler, B., Wang, Y., \& Coughlin, J. F. (2012). A field study on the impact of variations in short-term memory demands on drivers' visual attention and driving performance across three age groups. Human Factors, 54(3), 454-468.

33. Shutko, J., Mayer, K., Laansoo, E., \& Tijerina, L. (2009). Driver workload effects of cell phone, music player, and text messaging tasks with the Ford SYNC voice interface versus handheld visual-manual interfaces. 2009-01-0786.

34. Sodhi, M., Reimer, B., \& Llamazares, I. (2002). Glance analysis of driver eye movements to evaluate distraction. Behavior Research Methods, 34(4), 529538.

35. Thorslund, B., Ahlström, C., Peters, B., Eriksson, O., Lidestam, B., \& Lyxell, B. (2014). Cognitive workload and visual behavior in elderly drivers with hearing loss. European Transport Research Review, 6(4), 377-385. doi: 10.1007/s12544-014-0139-z

36. Tsimhoni, O., Yoo, H., \& Green, P. (1999). Effects of visual demand and in-vehicle task complexity on driving and task performance as assessed by visual occlusion. UMTRI-99-37.

37. Victor, T. (2005). Keeping eye and mind on the road.

38. Victor, T., Bärgman, J., Boda, C., et al. (2014). Analysis of Naturalistic Driving Study Data: Safer Glances, Driver Inattention, and Crash Risk: Safer.

39. Wang, Y., Reimer, B., Dobres, J., \& Mehler, B. (2014). The sensitivity of different methodologies for characterizing drivers' gaze concentration under increased cognitive demand. Transportation Research Part F, 26, 227-237.

40. Wierwille, W. W., Antin, J. F., Dingus, T. A., \& Hulse, M. C. (1988). Visual attentional demand of an in-car navigation display system. In Vision in Vehicles II. Second International Conference on Vision in Vehicles.

41. Wierwille, W. W., \& Tijerina, L. (1998). Modeling the relationship between driver in-vehicle visual demands and accident occurrence. Vision in Vehicles, 6, 233243. 